\title{
How do women feel about their cervical cytology samples being used for research?
}

\author{
Caroline Coope, ${ }^{1}$ Karen Rose, ${ }^{2}$ Anna Glasier, ${ }^{3}$ Judith Stephenson ${ }^{4}$
}

\begin{abstract}
'Research Fellow, Centre for Sexual Health \& HIV Research, Research Department of Infection and Population Health, University College London, London, UK

${ }^{2}$ Specialist Registrar, Well Woman Services, Dean Terrace Centre, Edinburgh, UK

${ }^{3}$ Honorary Professor, Department of Obstetrics \& Gynaecology,

The University of Edinburgh, Edinburgh and Faculty of Public Health and Policy, London School of Hygiene \& Tropical Medicine, London UK

${ }^{4}$ Margaret Pyke Professor of Sexual \& Reproductive Health Research Department of Reproductive Health, Institute for Women's Health, University College London, London, UK
\end{abstract}

\section{Correspondence to}

Dr Caroline Coope, Research Department of Epidemiology \& Public Health, University College London, 1-19 Torrington Place, London WC1E 6BT, UK;

caroline.coope@ucl.ac.uk

Received 20 December 2010

Accepted 17 May 2011

Published Online First

16 August 2011

\begin{abstract}
Background and methodology The introduction of liquid-based cytology (LBC) for cervical screening in the UK has enabled research into human papillomavirus as an adjunct to screening and provides potential opportunities for population-based research into women's health. The authors explored women's views on the use of remnant LBC samples for sexual and reproductive health research. A pilot study was conducted to assess the acceptability of collecting and storing remnant LBC specimens for future use in sexual and reproductive health research. Women attending a clinic for their routine smear test were recruited to the study. Semi-structured interviews were conducted with a subsample of the women to explore acceptability issues.
\end{abstract}

Results Of women invited to participate ( $n=369)$, $86 \%(n=316)$ consented to the storage and use of their remnant sample for research. Of these, $96 \%(n=304)$ consented to their sample being linked to reproductive health records. Women interviewed ( $n=23$ ) were happy with the research process although unclear about what future research might be conducted on their specimen and what health records would be accessed.

Discussion and conclusion As research becomes technically and ethically more complex the challenge remains to find the right balance between providing sufficient relevant information to ensure informed consent and allay participant's fears, while guarding against the inclusion of excessive detail. The introduction of LBC for cervical screening across the UK brings a new opportunity to integrate research studies within a national cervical screening programme. Our study suggests this is acceptable to women.

\section{Introduction}

Over the last decade, cervical screening in the UK has been transformed by replacing conventional 'Pap' smears with a new method of processing smears called liquid-based cytology (LBC). Change was swift in Scotland, where women aged 20-60 years old have been offered

\section{Key message points}

- Women attending a clinic for their routine cervical cytology test found the idea of storage and use of their leftover liquid-based cervical sample for research acceptable.

- Consenting women were not clear about what they had consented to in respect to possible future research studies and the health records that would be accessed.

- The consent process for the biobanking of residual cervical cytology samples for sexual and reproductive health research requires further research into the optimal approach to ensure 'informed' consent.

a cervical smear test using this method since 2003. In England, roll out of LBC to women aged 25-64 years started in 2004 as part of the National Health Service (NHS) Cervical Screening Programme. By the end of 2008 all laboratories had converted to LBC or had plans in place to convert in the near future.

After making the thin layer slide, there are typically enough cells remaining in the collection vial to carry out additional tests, including DNA-based nucleic acid amplification tests for sexually transmitted infections (STIs), such as Chlamydia trachomatis or Mycoplasma genitalium. Residual material from LBC samples has already been successfully used for molecular detection of human papillomavirus. ${ }^{1}$ Guidelines on the appropriate collection and storage of cervical cytology samples for biobanking purposes have more recently emerged, promoting this as a structural extension of current national screening and cancer registries. ${ }^{2} 3$ The introduction of wide-scale LBC offers an opportunity for repeated collection of cellular material from the lower genital tract of large numbers of women alongside their regular cervical screening. Researchorientated testing for other STIs or associated conditions (e.g. cervicitis, bacterial vaginosis) to improve understanding about the causes of sexual and reproductive ill health could be incorporated into this screening programme. For example, relatively little is known about the causes 
of pelvic inflammatory disease, ectopic pregnancy or tubal factor infertility. Clues to the aetiology of such conditions may lie in cervical material that can be accessed through remnant LBC samples.

In theory, remnant LBC samples can be stored, tested at a later date, and the test results linked to reproductive health care records, to test hypotheses about the causes of conditions such as ectopic pregnancy, tubal factor infertility, recurrent spontaneous abortion and preterm birth. A number of challenges need to be overcome for such studies to be conducted related to the collection of human tissue samples for analysis at a future date and the measurement of as yet unspecified substances, as well as linking these data to health care records, which raises complex ethical and practical issues. A one-time general consent for research on biological samples is supported by empirical findings as the best approach to such studies. ${ }^{4}$ Public attitudes to donating leftover samples from routine clinical tests suggest the majority would consider giving open-ended consent for storage and later use in research. ${ }^{5}$ However, empirical evidence is lacking in respect to specific studies for sexual and reproductive health research. In view of this need for evidence, a pilot study was commissioned to explore the acceptability of using LBC remnant samples for sexual and reproductive health research. Our aim was to explore women's views and understanding of 'onetime consent' for using residual material from their LBC samples for future biomedical research including tests for STIs and linking this to health service records on pregnancy. Since the concept of donating a sample of tissue for unspecified research is complex, we wanted to explore the reasons why women consented to sexual and reproductive health research, their views on the adequacy of the research information and their understanding of what they had consented to. We also wanted to assess whether women would consent to the linkage of their remnant sample to reproductive health records and explore women's understanding of this.

\section{Methods}

\section{Sample selection and recruitment}

The study was conducted in a large Family Planning/ Well Woman Clinic in Scotland. All women attending the clinic for a routine cervical smear test were invited to take part. On arrival at the clinic women were given a participant information sheet (PIS) that explained the research, and were asked to read this while they waited to be seen. The PIS contained the required information stipulated by the NHS National Research Ethics Service guidelines, an excerpt of which is shown in Box 1.

Women attended their clinic appointment as usual. After their cervical sample had been taken, the clinician invited them to take part in the study. Consent forms were attached to cervical cytology request forms as an aide memoire for clinicians. Women who declined to take part were asked the reason for this which, if given, was recorded on the blank consent form.

A sample size of 369 women was calculated to estimate the acceptability (consent) rate with reasonable precision. For example, for $80 \%$ of women giving consent the $95 \%$ CI would be $76 \%$ to $84 \%$ and for $50 \%$ consent it would be $45 \%$ to $55 \%$. Recruitment took place over a 2 -month period.

\section{Data collection and analysis}

Consent consisted of three parts including consent to their leftover LBC sample being stored and used for later research, the use of their personal information collected on the cytology request form, and their specimen being linked to their health service records on pregnancy.

Consent responses and information from cervical cytology request forms were entered into an Excel database at the research site and later transferred to Stata/SE Version 11.0 (Stata Corp, College Station, TX, USA) for further analysis.

Semi-structured interviews were conducted on four separate clinic days when women were additionally invited to take part in a short interview. The semistructured interviews were conducted using a topic guide $^{6}$ with prompts to obtain participants' views on the information given about the study; their understanding of what they had consented to; their reasons for consenting or not; how they felt about their sample being tested for STIs; their understanding of what it meant for samples to be linked to health service records on pregnancy; and their thoughts about the confidentiality of the research. All interviews were conducted by the same researcher, in a private room within the clinic, and were recorded and later transcribed, for which separate consent was sought. Interview transcripts were analysed using a thematic approach and key themes and concerns drawn out. ${ }^{7}$ The total number of interviews $(n=23)$ achieved adequacy, so that sufficient data were collected for saturation to occur and variations in the data were explored and accounted for. ${ }^{8}$

\section{Box 1 Excerpt from the patient information}

\section{sheet}

"The purpose of the study is to find out whether women who come for a routine cervical smear test would be happy for their leftover screening sample to be used for research into women's reproductive health. With the new method of cervical screening that is used in this clinic (called liquid-based cytology, or LBC) it is possible to do other tests on the remains of your sample after checking for cervical disease. We would like to use LBC samples to look for infections or other factors that might put women at higher risk of fertility problems or premature childbirth. To do this, we would need to link up your LBC sample with your Health Service records regarding any pregnancy. You would not be asked to provide any extra information or any extra samples to the research team. Several studies with different tests could be done using stored LBC samples. Each study would be reviewed in detail by a research ethics committee before it could go ahead." 


\section{Results}

\section{Consent rates}

Of the 369 women recruited to the study, $86 \%(n=316)$ consented to their leftover LBC sample being stored and used for later research. Of these, 96\% $(n=304)$ additionally consented to both the use of their personal information collected on the cytology request form and their specimen being linked to their health service records on pregnancy; $3 \%(n=10)$ to the use of their personal data but no specimen-record linkage; and $1 \%(n=2)$ to their specimen being linked to their health service records on pregnancy but no use of personal data. Health information of the consenting women obtained from the cervical cytology request forms are presented in Table 1. Consenting women ranged in age from 20 to 61 years, with the majority (79\%) being within the age range 20-39 years. A similar age distribution was found in the women interviewed.

\section{Reasons for declining consent}

Reasons given by women who declined to take part $(n=53)$ included: not receiving an information leaflet at reception $(n=20)$; not having enough time to consider the invitation $(n=6)$; being unable to read the information leaflet due to limited English language or sight difficulties $(n=2)$; and not wanting health records on pregnancy accessed because of their content $(n=1)$. No reason was recorded for the remaining women.

Table 1 Distribution of reproductive characteristics of the study sample by consent type $(n=316)$ and interview conducted $\left(n=23^{*}\right)$

\begin{tabular}{|c|c|c|c|c|c|}
\hline \multirow[b]{2}{*}{ Characteristic } & \multirow[b]{2}{*}{$\begin{array}{l}\text { Total sample } \\
\text { (n) }\end{array}$} & \multicolumn{3}{|c|}{ Consent type $[n(\%)]$} & \multirow[b]{2}{*}{$\begin{array}{l}\text { Interview } \\
\text { conducted [n(\%)] }\end{array}$} \\
\hline & & $\begin{array}{l}\text { Specimen, data } \\
\text { and linkage }\end{array}$ & $\begin{array}{l}\text { Specimen and } \\
\text { data }\end{array}$ & $\begin{array}{l}\text { Specimen and } \\
\text { linkage }\end{array}$ & \\
\hline \multicolumn{6}{|l|}{ Age group (years) } \\
\hline $20-29$ & 139 & $138(45)$ & $1(10)$ & 0 & $10(44)$ \\
\hline $30-39$ & 109 & $102(34)$ & $7(70)$ & 0 & $7(30)$ \\
\hline $40-49$ & 47 & $46(15)$ & $1(10)$ & 0 & $4(17)$ \\
\hline $50+$ & 16 & $16(5)$ & 0 & 0 & $1(4.5)$ \\
\hline No data & 5 & $2(1)$ & $1(10)$ & $2(100)$ & $1(4.5)$ \\
\hline \multicolumn{6}{|l|}{ Indications for sample } \\
\hline Routine & 227 & $221(73)$ & $6(60)$ & 0 & $20(86.5)$ \\
\hline Early recall & 83 & $79(26)$ & $4(40)$ & 0 & $3(12.5)$ \\
\hline Annual recall & 1 & $1(0.25)$ & 0 & 0 & 0 \\
\hline No data & 5 & $3(0.75)$ & 0 & $2(100)$ & 0 \\
\hline \multicolumn{6}{|l|}{ Method of contraception } \\
\hline Oral contraceptive & 119 & $116(38)$ & $3(30)$ & 0 & $7(30)$ \\
\hline Intrauterine device & 29 & $28(9)$ & $1(10)$ & 0 & $2(9)$ \\
\hline Female/male barrier & 60 & $57(19)$ & $3(30)$ & 0 & $7(30)$ \\
\hline Female/male sterilisation & 12 & $11(3.5)$ & $1(10)$ & 0 & $1(4.5)$ \\
\hline Implanon ${ }^{\circledR}$ & 8 & $8(3)$ & 0 & 0 & 0 \\
\hline Other & 16 & $15(5)$ & $1(10)$ & 0 & 0 \\
\hline None & 69 & $68(22)$ & $1(10)$ & 0 & $6(26.5)$ \\
\hline No data & 3 & $1(0.5)$ & 0 & $2(100)$ & 0 \\
\hline \multicolumn{6}{|l|}{ Births (live and still) (n) } \\
\hline 0 & 233 & $227(75)$ & $6(60)$ & 0 & $17(74)$ \\
\hline 1 & 23 & $22(7)$ & $1(10)$ & 0 & $2(9)$ \\
\hline 2 & 24 & $24(8)$ & 0 & 0 & $3(12.5)$ \\
\hline 3 & 6 & $6(2)$ & 0 & 0 & 0 \\
\hline 4 & 5 & $4(1)$ & $1(10)$ & 0 & 0 \\
\hline No data & 25 & $21(7)$ & $2(20)$ & $2(100)$ & $1(4.5)$ \\
\hline \multicolumn{6}{|c|}{ Abortions (ectopic, spontaneous abortion) ( $n$ ) } \\
\hline 0 & 225 & $218(72)$ & $7(70)$ & 0 & $21(91)$ \\
\hline 1 & 44 & $44(14)$ & 0 & 0 & $1(4.5)$ \\
\hline 2 & 10 & $10(3)$ & 0 & 0 & 0 \\
\hline 3 & 2 & $2(1)$ & 0 & 0 & 0 \\
\hline No data & 35 & $30(10)$ & $3(30)$ & $2(100)$ & $1(4.5)$ \\
\hline
\end{tabular}




\section{Semi-structured interviews}

Twenty-six women were invited to take part in a short interview following their cervical cytology appointment, of whom $89 \%(n=23)$ agreed. The short semistructured interviews were conducted immediately after the participant's clinic appointment with the objective of capturing the woman's first-hand experience of the research process and her immediate understanding of what she had consented to and any concerns this may have raised about the research. Twenty-one (91\%) of the women interviewed had consented to all three parts of the study. Further details about the interviewees can be found in Table 1 .

Women felt they had had sufficient time to read the PIS prior to their appointment and all agreed the information sheet was clear, understandable and "not too long". During later questions about the research a number of the interviewees said they had only "skim" read the PIS and hence could not recall details of the study. Women were asked if they recalled any questions they had about the research after initially being asked to participate by their clinician. Questions they recalled included: "Who was conducting the research?", "Was the research only about fertility and pregnancy?" and "Was it only for women who had ever been pregnant?".

Women were asked what they understood by their consent in respect to each part of the consent. For part one of the consent, most women understood that following their smear test the leftover cells would be stored and used for further research. One woman thought only the results of the cytological test would be kept, along with subsequent ones. Part two of the consent, concerning the use of their details from the cytology request forms, was clearly understood by all the interviewees. Part three of the consent, which asked for agreement to link their leftover LBC sample to their health service records on pregnancy, was the least well-understood aspect of consent. Despite having consented to part three, women were unclear about what "health service records on pregnancy" actually meant. When possible options were proposed such as hospital and general practitioner (GP) records some women were clearly concerned about researchers accessing GP records. Women who said they were not concerned about their GP records being accessed added this was because "there is nothing in them anyway". One woman thought she had consented to access of records kept at the clinic only.

Women expressed three main reasons why they had consented to take part in the research. First, that the research required no "extra activity" beyond consenting to take part, after having their normal smear test. Second, women were very keen to support reproductive health research and a number felt there was a great need to advance knowledge in this area. Third, women felt a desire to help other women in respect to infertility with a few disclosing their own, or family and friends' fertility problems as the motivation to take part. Women were clearly aware that taking part in the research would not directly benefit them, but hoped it might impact on future treatments, knowledge and services for women.

Women were asked how they would feel if their remnant sample was tested for STIs. Responses ranged from having no concern, to being troubled about this. The PIS stated "we would like to use LBC samples to look for infections or other factors", however some women felt they had not consented specifically to tests for STIs. Women raised the issue of whether they would be informed of the results if such tests were conducted, with most saying they would expect to be informed.

Lastly, women were asked whether they felt assured about the confidentiality of the study. Women unanimously agreed that the explanations given about confidentiality had reassured them of this.

\section{Discussion}

There was a fairly high acceptability rate among women invited to take part in the study, in particular for consent to their leftover LBC sample being processed and stored for research (86\%). Other studies that have used remnant LBC (e.g. for comparing cervical cancer detection methods) achieved higher consent rates (92\%). ${ }^{9}$ This may be partly due to the unequivocal nature of the studies compared with our request for open-ended consent for potentially multiple studies. It is also possible that our target population, which was recruited from a specialist clinic, may represent a slightly higherrisk group compared with a general practice population, which may account for the lower consent rate.

The consent rates in our study support a 'once for all' consent approach to the collection and storage of remnant LBC samples for future research, with the proviso that an ethics committee would independently review each future study. Indeed a major reason for taking part in the study was that it involved no further activity by them. However, on further probing during semi-structured interviews it became clear that under certain circumstances women expected they would be re-contacted, for instance if their sample tested positive for a treatable STI. Under these conditions women expected two things: first, that they would receive specific information about the tests and, second, that they would be informed if they had positive tests for treatable infections. This poses the problem that if samples are unlinked and anonymised then informing individuals of test results would be impossible. Under these circumstances, as with the Biobank project, participants would need to understand that they could not receive certain test results, but that they could undergo named, voluntary testing through other health care channels.

Slightly fewer women consented to have their sample linked to their health service records on pregnancy. This was expected and in line with other research on 
consent to storage and future research using routinely collected human tissue samples. ${ }^{10}$ Consultations conducted in preparation for the UK Biobank similarly found people felt uneasy about their health records being accessed for research purposes. ${ }^{11}$ Women interviewed admitted they were not clear what records their sample would be linked to. It seems likely that efforts made by researchers to simplify the language used in the consent documentation may have resulted in increased vagueness rather than clarity. However, prior research has shown that people's fears can be allayed if explanations of why information would be helpful to researchers are made clear and safeguards against unauthorised access are given. ${ }^{11}{ }^{12} \mathrm{~A}$ key challenge faced by those conducting such research is to find the right balance between providing sufficient relevant information to ensure informed consent and to allay participant's fears, while guarding against the inclusion of excessive detail. ${ }^{12}$ One solution is to provide a limited amount of accurate and relevant information in the first instance, combined with a user-friendly way for participants to access extended documents allowing them to check more detailed and technical information. Another option could be to provide a summary information sheet together with a more detailed sheet for participants to read at their leisure.

A different option is the use of human tissue samples without consent. The UK Human Tissues Act (2004) states "consent is not required to use tissue obtained from living patients if the tissue is anonymous to the researcher and the project has research ethics approval". ${ }^{13}$ Further studies are needed to explore this research without consent option within the context of sexual and reproductive health.

\section{Conclusion}

With the introduction of LBC for cervical screening across the UK comes an unprecedented opportunity to integrate research studies within a national cervical screening programme. Our preliminary research suggests this is acceptable to women although further research to explore feasibility issues is required.

Acknowledgements The authors thank Dr Alistair Williams, Consultant Pathologist, Department of Pathology, Royal Infirmary of Edinburgh, and staff in the pathology laboratory who isolated, stored and transferred specimens; staff of NHS Lothian Family Planning and Well Woman for recruiting women to the study; Jeremy Ford, Laboratory Manager, Tumour Marker Laboratory, UCL, for storage of the specimens; Dr Caroline Carder, UCH NHS Foundation Trust, Chlamydia Laboratory, for storage and analysis of specimens; and Heidi Chandler for preparation of research documents and transcription of interviews.

Funding This work was undertaken at UCLH/UCL who received a proportion of funding from the
Department of Health's National Institute for Health Research Centres funding scheme. The authors are also grateful for the support of the Margaret Pyke Trust for their ongoing contribution to the programme of reproductive health research at UCL. The study was funded by UCLH Clinical Research Development Committee. The funding source was responsible for the London site ethical review of study. They had no other involvement in the study.

Competing interests None.

Ethical approval The study was ethically reviewed and approved by UCLH Clinical Research Development Committee and NHS Lothian.

Provenance and peer review Not

commissioned; externally peer reviewed.

\section{References}

1 Cuschieri KS, Cubie HA, Whitley MW, et al. Multiple high risk HPV infections are common in cervical neoplasia and young women in a cervical screening population. J Clin Pathol 2004;57:68-72.

2 Arbyn M, Van Veen EB, Andersson K, et al. Cervical cytology biobanking in Europe. Int J Biol Markers 2010;25:117-125.

3 Arbyn M, Andersson K, Bergeron C, et al. Cervical cytology biobanks as a resource for molecular epidemiology. Methods Mol Biol 2011;675:279-298.

4 Wendler D. One-time general consent for research on biological samples. BMJ 2006;332:544-547.

5 Treweek S, Doney A, Leiman D. Public attitudes to the storage of blood left over from routine general practice tests and its use in research. J Health Serv Res Policy 2009;14:13-19.

6 Arthur S, Nazroo J. Designing fieldwork strategies and materials. In: Ritchie J, Lewis J (eds), Qualitative Research Practice: A Guide for Social Science Students and Researchers. London, UK: Sage Publications, 2003;109-137.

7 Ritchie J, Spencer L, O'Connor W. Carrying out qualitative analysis. In: Ritchie J, Lewis J (eds), Qualitative Research Practice: A Guide for Social Science Students and Researchers. London, UK: Sage Publications, 2003;219-262.

8 Flick U. An Introduction to Qualitative Research (3rd edn). Thousand Oaks, CA: Sage Publications, 2006.

9 Bigras G, de Marval F. The probability for a Pap test to be abnormal is directly proportional to HPV viral load: results from a Swiss study comparing HPV testing and liquid-based cytology to detect cervical cancer precursors in 13,842 women. Br J Cancer 2005;93:575-581.

10 Barbour V. UK Biobank: a project in search of a protocol? Lancet 2003;361:1734-1738.

11 The Wellcome Trust and Medical Research Council. Public Perceptions of the Collection of Human Biological Samples. 2000. http://www.ukbiobank.ac.uk/docs/perceptions.pdf [accessed 12 January 2007].

12 O'Neill O. Some limits of informed consent. J Med Ethics 2003;29:4-7.

13 Human Tissue Authority. Code of Practice 9: Research Section: Human Tissue and Research. 2009. http://www.hta. gov.uk/legislationpoliciesandcodesofpractice/codesofpractice/ code9research.cfm?FaArea1 = customwidgets.content_ view_1\&cit_id=762\&cit_parent_cit_id=757 [accessed 29 March 2011]. 